\title{
Relationships between Iraqi Rice Varieties at the Nuclear and Plastid Genome Levels
}

\author{
Hayba Badro $\mathbb{D}$, Agnelo Furtado and Robert Henry * $\mathbb{D}$ \\ Queensland Alliance for Agriculture and Food Innovation, University of Queensland, Brisbane, QLD 4072, \\ Australia; haybaq@yahoo.com (H.B.); a.furtado@uq.edu.au (A.F.) \\ * Correspondence: robert.henry@uq.edu.au
}

Received: 16 September 2019; Accepted: 5 November 2019; Published: 7 November 2019

\begin{abstract}
Due to the importance of the rice crop in Iraq, this study was conducted to determine the origin of the major varieties and understand the evolutionary relationships between Iraqi rice varieties and other Asian rice accessions that could be significant in the improvement of this crop. Five varieties of Oryza sativa were obtained from Baghdad/Iraq, and the whole genomic DNA was sequenced, among these varieties, Amber33, Furat, Yasmin, Buhooth1 and Amber al-Baraka. Raw sequence reads of 33 domesticated Asian rice accessions were obtained from the Sequence Read Archive (SRA-NCBI). The sequence of the whole chloroplast-genome was assembled while only the sequence of 916 concatenated nuclear-genes was assembled. The phylogenetic analysis of both chloroplast and nuclear genomes showed that two main clusters, Indica and Japonica, and further five sub-clusters based upon their ecotype, indica, aus, tropical-japonica, temperate-japonica and basmati were created; moreover, Amber33, Furat, Yasmin and Buhooth1 belonged to the basmati, indica and japonica ecotypes, respectively, where Amber33 was placed in the basmati group as a sister of cultivars from Pakistan and India. This confirms the traditional story that Amber was transferred by a group of people who had migrated from India and settled in southern Iraq a long time ago.
\end{abstract}

Keywords: rice (Oryza sativa); evolutionary relationships; chloroplast genome; nuclear genome; phylogeny

\section{Introduction}

Rice is grown in a wide range of environments worldwide, however, most of the world's rice is cultivated and consumed in Asia [1-3]. Iraq has favorable agricultural conditions for rice cultivation, where rice is a staple food for the majority of the Iraqi people [4]. In Iraq, rice grows as a summer crop, and there are a number of traditional, introduced and improved rice varieties that are cultivated in the central and southern region, as well as in the valleys of northern Iraq [1].

The variety Amber is the most important local Iraqi rice variety and is characterised by high quality in terms of taste (aromatic character) [1]. It has been cultivated in central and southern Iraq, especially in the marshes, for a long time. Anecdotal evidence suggests that Amber was introduced to the marshlands of southern Iraq when water buffalo breeding was introduced to the region by a foreign group from the south Asia, probably from the Indian subcontinent. This popular view was reported in a study by Al-Zahery et al. [5], that highlighted the paternal and maternal origin of the human population in the marsh areas, and observed marginal influences of Indian origin on the gene pool of an autochthonous population of the region. A number of rice varieties have also been introduced to Iraq since the middle of the last century to improve rice productivity [6]. IR8 was the first variety introduced in 1968 by the International Rice Research Institute (IRRI) (Philippines), it has high yield potential but the grain quality has not been high compared to Amber. Since aroma is one of the key traits in determining grain quality in rice [7], Amber became a control variety in the central 
and south regions of Iraq to assess the grain quality of introduced varieties [1]. Accordingly, Furat and Yasmin were also introduced from Vietnam to Iraq in the late 20th century because they are aromatic, tolerant to limited water, highly productive, and have high grain quality [4]. An understanding of the origin of local Iraqi rice and the genetic relationships between Iraqi rice and Asian domesticated rice will effectively guide Iraqi rice breeding (the aim of the current study). However, few studies have investigated Iraqi rice in general, and the origin and the evolution of Iraqi varieties, especially Amber, in particular $[4,8,9]$.

Each living organism is the consequence of an evolutionary process [10]; therefore, it is imperative to enrich our perception of the evolutionary history of organisms and the relationships among them to guide their genetic improvement. Methods of determining evolutionary history (Phylogeny) have undergone many stages of development. Morphological markers maybe influenced by environmental factors and growth practices. More recent methods have used molecular markers which are independent of environmental factors [11], including techniques such as RFLP, AFLP, RAPD, SSR and ISSR along with morphological markers, to study phylogenetic relationships [12]. The development of high-throughput sequencing technology has revolutionised the study of genetics and evolutionary relationships. Most recently, through next-generation sequencing (NGS), whole-genome sequencing and re-sequencing have become available, so the investigation of the entire genome, rather than targeting precise regions, is now a real opportunity [13-15].

Every plant cell has three genomes—nuclear, chloroplast, and mitochondrial—-that may differ in evolutionary history. The chloroplast genome is a maternal genome which is highly-conserved and not involved in recombination, therefore, it is the most commonly used tool to determine the origin and the evolutionary relationships among plant species [16-19]. However, sometimes, evolutionary analysis based on the chloroplast genome must be supported by nuclear genome-based analysis to achieve the most reliable results because the chloroplast genome can only represent the maternal evolutionary history with a slow evolutionary rate $[20,21]$. Phylogenetic analysis using the nuclear genome can deliver inconsistent trees due to recombination that may confuse phylogenetic resolution. However, this analysis provides greater insights into evolutionary relationships. Several studies have strongly suggested applying this analysis along with chloroplast phylogenetic analysis $[18,19,22]$. Many studies have applied phylogenetic analysis at both genome levels [23-25], and the results of most of these studies showed that the nuclear genome followed a different evolutionary history pattern to that of the chloroplast genome.

We reported the whole chloroplast genome sequences for Iraqi rice and compared them with the whole chloroplast sequences of other domesticated Asian rice varieties. This provided an important tool for estimating genetic distance and determining evolutionary relationships between rice accessions; the nuclear genomes also provided further information on the relationships between the varieties studied. The study aimed to determine the origin and evolution of Iraqi rice, especially Amber33.

\section{Results}

\subsection{DNA Sequencing and Data Processing}

The sequencing process of the five Iraqi varieties (Table 1 ) generated about $51 \mathrm{~Gb}$ of data containing 337 million of 151-bp paired-end reads. The minimum and the maximum number of reads were about 58 and 93 million reads with sequence depth ranging between $23 \times$ and $38 \times$ for Buhooth 1 and Furat, in turn. When raw data was trimmed at the quality limit of 0.01 , an average of $15 \%$ of the reads' length and $9 \%$ of the number of reads were removed, thus the number of reads and data coverage reduced to the range between 53 and 86 million, and $18 \times$ and $30 \times$, respectively (Table S1). In terms of downloaded data (Table 2), the average length of raw reads was 83-bp, and the minimum and the maximum number of reads ranged between 43 and 117 million reads while the sequence coverage fluctuated between $10 \times$ and $26 \times$. Finally, the number of reads and the data coverage of each of the data sets were assessed after trimming the raw reads at the quality limit of 0.01 (Table S1). 
Table 1. The Iraqi plant materials used in this study.

\begin{tabular}{ccccc}
\hline Varieties & History & Varietal Group & BioProject ID & $\begin{array}{c}\text { BioSample } \\
\text { Accessions }\end{array}$ \\
\hline Amber33 & $\begin{array}{c}\text { Local (Iraq) } \\
\text { Introduced from } \\
\text { (Vietnam) in 1996 }\end{array}$ & Aromatic, medium grain type & PRJNA576935 & SAMN13014963 \\
Furat & $\begin{array}{c}\text { Antroduced from } \\
\text { (Vietnam) in 1998 }\end{array}$ & Aromatic, medium grain type & PRJNA576935 & SAMN13014964 \\
Yasmin & Improved & Non-Aromatic, long grain type & PRJNA576935 & SAMN13014965 \\
Buhooth1 & Improved & Aromatic, long grain type & PRJNA576935 & SAMN13014966 \\
Amber al-Baraka & & & SAMN13014967 \\
\hline
\end{tabular}

Table 2. Summary of data downloaded for sequence comparisons.

\begin{tabular}{|c|c|c|c|c|c|c|}
\hline No & Sample Unique ID & $\begin{array}{c}\text { Project } \\
\text { Accession }\end{array}$ & Species & $\begin{array}{l}\text { Country of } \\
\text { Origin }\end{array}$ & Ecotype * & $\begin{array}{c}\text { Alignment Name } \\
\text { (in Figures } 1 \text { and } 2) \text { * }\end{array}$ \\
\hline 1 & B243 & ERP005654 & O. sativa & China & Aus & $\mathrm{Ch}$ (Aus)B243 \\
\hline 2 & CX165 & ERP005654 & O. sativa & China & TmpJ & Ch(TmpJ)CX165 \\
\hline 4 & CX10 & ERP005654 & O. sativa & China & In & $\mathrm{Ch}(\mathrm{In}) \mathrm{CX} 10$ \\
\hline 5 & CX368 & ERP005654 & O. sativa & India & Aus & India(Aus)CX368 \\
\hline 6 & IRIS_313-10670 & ERP005654 & O. sativa & India & Bas & India(Bas)IRIS_313-10670 \\
\hline 9 & IRIS_313-11152 & ERP005654 & O. sativa & India & In & India(In)IRIS_313-11152 \\
\hline 10 & CX129 & ERP005654 & O. sativa & Indonesia & TrpJ & Indo(TrpJ)CX129 \\
\hline 11 & CX25 & ERP005654 & O. sativa & Indonesia & In & Indo(In)CX25 \\
\hline 12 & CX104 & ERP005654 & O. sativa & Iran & Bas & Iran(Bas)CX104 \\
\hline 13 & CX227 & ERP005654 & O. sativa & Japan & Aus & Jap(Aus)CX227 \\
\hline 18 & IRIS_313-11026 & ERP005654 & O. sativa & Pakistan & Bas & Pak(Bas)IRIS_313-11026 \\
\hline 19 & IRIS_313-8656 & ERP005654 & O. sativa & Pakistan & Bas & Pak(Bas)IRIS_313-8656 \\
\hline 20 & IRIS_313-11829 & ERP005654 & O. sativa & Pakistan & TmpJ & Pak(TmpJ)IRIS_313-11829 \\
\hline 21 & IRIS_313-10380 & ERP005654 & O. sativa & Philippines & Aus & Phil(Aus)IRIS_313-10380 \\
\hline 22 & CX59 & ERP005654 & O. sativa & Philippines & Bas & Phil(Bas)CX59 \\
\hline 23 & IRIS_313-10373 & ERP005654 & O. sativa & Philippines & TmpJ & Phil(TmpJ)IRIS_313-10373 \\
\hline 24 & $\mathrm{C} \times 243$ & ERP005654 & O. sativa & Philippines & TrpJ & Phil(TrpJ)C $\times 243$ \\
\hline 25 & IRIS_313-9505 & ERP005654 & O. sativa & Philippines & In & Phil(In)IRIS_313-9505 \\
\hline 26 & CX126 & ERP005654 & O. sativa & Philippines & In & Phil(In) $\bar{C} \times 126$ \\
\hline 27 & IRIS_313-10718 & ERP005654 & O. sativa & Sri Lanka & Aus & SriL(Aus)IRIS_313-10718 \\
\hline 28 & IRIS_313-9949 & ERP005654 & O. sativa & Sri Lanka & TrpJ & Sril(TrpJ)IRIS_313-9949 \\
\hline 29 & IRIS_313-11248 & ERP005654 & O. sativa & Thailand & TrpJ & Thai(TrpJ)IRIS_313-11248 \\
\hline
\end{tabular}

32 domesticated Asian rice accessions and one domesticated African rice as an out-group downloaded from SAR-NCBI: their unique ID, species, country of origin, and ecotype was from the study of [26]; the alignment names were generated in this study. ${ }^{*}$ In: indica subpopulation, TrpJ: tropical japonica subpopulation, TmpJ: temperate japonica subpopulation, Aus: aus population, Bas: basmati population, Ch: China, Indo: Indonesia, Jap: Japan, Pak: Pakistan, Phil: Philippines, Sril: SriLanka, Viet: Vietnam.

\subsection{Chloroplast Genome Assembly}

Mapping all varieties against the reference, O. sativa sub sp. japonica Nipponbare "GenBank: GU592207.1", under three various fraction settings clarified the most accurate and reliable mapping setting. The number of mismatches and gaps of each variety was virtually stable in all different settings (Table S2). Indeed, this stability confirms that most of these variations were produced from actual differences between the sequences of samples and reference, not due to using different settings; based on that, setting number two (length fraction (LF) of 1 and similarity fraction (SF) of 0.8 ) was applied to the other steps of assembly, Improvement process (Imp). Moreover, three different settings of Word " $\mathrm{W}$ " and Bubble " $\mathrm{B}$ " size in de novo assembly generated a satisfactory number of contigs that cover the whole chloroplast genome area, around five large chloroplast-contigs with a length of more than $12 \mathrm{~kb}$ produced from each setting. Subsequently, four main regions of the chloroplasts, large single copy 
(LSC), inverted repeat A (IR A), small single copy (SSC) and inverted repeat B (IR B), were assembled successfully for all 38 varieties through a de novo assembly pipeline. The lengths of these regions were about $80 \mathrm{~kb}$ for LSC, $12 \mathrm{~kb}$ for SSC and $20 \mathrm{~kb}$ for IR A and IR B (Figure S1 shows only Iraqi rice varieties). In manual-curation, the comparison between both sub-approaches of the chloroplast genome assembly pipeline showed no significant differences in terms of the number of variations; however, any minor conflicts were resolved by reference to the reads (Table S3 shows only Iraqi rice varieties). The minimum and maximum lengths of the whole chloroplast for all Iraqi varieties and downloaded accessions were 134,259 and 134,556 bp, respectively, while the coverages ranged from $839 \times$ to up to $11,466 \times$, and the average coverage was $3818 \times$ (Table 3$)$.

Table 3. The results of the chloroplast and nuclear genome assembly.

\begin{tabular}{|c|c|c|c|}
\hline \multirow{2}{*}{ Varieties } & \multicolumn{2}{|c|}{ Chloroplast Genome } & \multirow{2}{*}{$\begin{array}{l}\text { Length of Nuclear } \\
\text { Genome (bp) }\end{array}$} \\
\hline & Length of Genome (bp) & Coverage $(\times)$ & \\
\hline Amber33 & 134,536 & 2909 & 616,371 \\
\hline Furat & 134,500 & 7819 & 616,190 \\
\hline Yasmin & 134,502 & 5495 & 616,301 \\
\hline Buhooth1 & 134,550 & 4759 & 616,393 \\
\hline Amber al-Baraka & 134,493 & 3651 & 616,310 \\
\hline B243 & 134,497 & 2203 & 616,278 \\
\hline CX165 & 134,542 & 8818 & 616,377 \\
\hline CX352 & 134,553 & 5305 & 616,324 \\
\hline CX10 & 134,503 & 11,466 & 616,274 \\
\hline CX368 & 134,504 & 3674 & 616,236 \\
\hline IRIS_313-10670 & 134,535 & 1669 & 616,369 \\
\hline IRIS_313-11153 & 134,551 & 1639 & 616,360 \\
\hline IRIS_313-11479 & 134,259 & 2726 & 616,367 \\
\hline IRIS_313-11152 & 134,503 & 1413 & 616,271 \\
\hline CX129 & 134,535 & 6076 & 616337 \\
\hline $\mathrm{CX} 25$ & 134,503 & 5830 & 616,210 \\
\hline CX104 & 134,532 & 6784 & 616,348 \\
\hline CX227 & 134,504 & 4267 & 616,314 \\
\hline CX140 & 134,547 & 5185 & 616,393 \\
\hline IRIS_313-10073 & 134,556 & 2036 & 616,355 \\
\hline IRIS_313-10549 & 134,495 & 1636 & 616324 \\
\hline IRIS_313-11021 & 134,531 & 1978 & 616,383 \\
\hline IRIS_313-11026 & 134,532 & 1723 & 616,358 \\
\hline IRIS_313-8656 & 134,532 & 2334 & 616380 \\
\hline IRIS_313-11829 & 134,539 & 4164 & 616,389 \\
\hline IRIS_313-10380 & 134,496 & 1857 & 616,331 \\
\hline CX59 & 134,536 & 5913 & 616,370 \\
\hline IRIS_313-10373 & 134,551 & 1464 & 616,363 \\
\hline CX243 & 134,556 & 4375 & 616,362 \\
\hline IRIS_313-9505 & 134,503 & 968 & 616,283 \\
\hline$\overline{\mathrm{C}} \times 126$ & 134,503 & 3510 & 616,220 \\
\hline IRIS_313-10718 & 134,531 & 2332 & 616,324 \\
\hline IRIS_313-9949 & 134,532 & 3041 & 616385 \\
\hline IRIS_313-11248 & 134,413 & 974 & 616,336 \\
\hline CX106 & 134,529 & 6145 & 616,339 \\
\hline B009 & 134,528 & 839 & 616,231 \\
\hline $\mathrm{CX} 37$ & 134,503 & 4836 & 616,258 \\
\hline O.glaberrima-PRJNA13765 & 134,542 & 2567 & 616,099 \\
\hline
\end{tabular}

The table includes the length of the chloroplast genome, the number of bases of mapped reads, and the coverage of assembled chloroplast genome for five Iraqi varieties and 32 domesticated Asian accessions and one domesticated African rice as an out-group downloaded from SAR-NCBI. This table also shows the length of the nuclear genome. 


\subsection{Phylogenetic Analysis of the Chloroplast Genome}

Two phylogenetic approaches were used to analyse the multiple alignments of thirty-nine chloroplast genomes which had a total length of $134,535 \mathrm{bp}$. Although the result of both phylogenetic methods showed some minor alterations at the end of some subclades, the content of the main clades and subclades, which followed their ecotype classifications, were identical (Figure 1). Phylogenetic analysis of the chloroplast genome divided the thirty-nine rice accessions into two main clades, an Indica clade and a Japonica clade. The Indica clade (In) included most individuals under indica (6 accessions) and aus (5 accessions) ecotypes except two individuals, B009 and IRIS_313-10718. The Japonica clade contained two subclades, a main Japonica clade and a Basmati clade; the first subclade which was the main Japonica clade (Jap) included all japonica individuals (13 accessions) from the two subpopulations of japonica ecotype, tropical and temperate, while the second subclade, the Basmati clade (Bas), involved all individuals of basmati ecotype (6 accessions) and the excluded individuals from the first clade (Indica). Additionally, the Iraqi varieties were distributed as following: Furat, Yasmin and Amber al-Baraka into the Indica clade whereas Amber33 and Buhooth1 into the Japonica clade. Buhooth1 was close to accessions from tropical japonica ecotype more than accessions under temperate japonica ecotype, and interestingly, Amber33 was located within the Basmati subclade.

The multiple alignments of chloroplast genomes comprised $134,535 \mathrm{bp}$, the number of identical sites was 134,270 characters (99.8\%) while the number of variable bases among all the accessions totaled $265(0.2 \%)$. These 265 variable bases were sorted into 85 variation positions which were in turn grouped into four types of polymorphisms including single nucleotide polymorphism (SNP), multi nucleotide polymorphism (MNP), insertions (Ins) and deletions (Del) (Table 4). The most abundant polymorphism types among all accessions were SNPs. Out of 85 polymorphisms, 83\%, 12\% and 5\% were located in the four main regions of the chloroplast genome, LSC, SSC and IR A and B, respectively (Table S4).

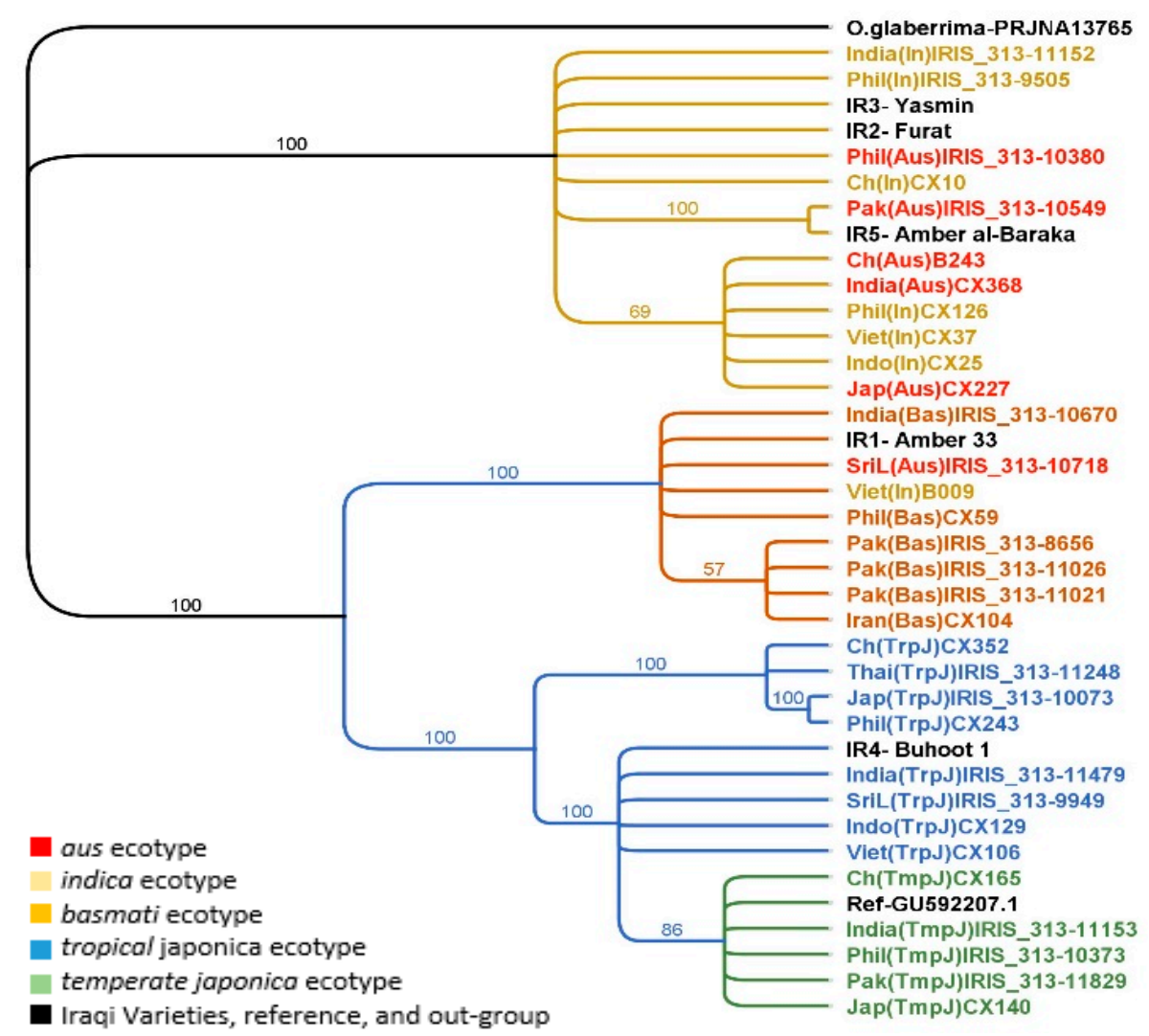

Figure 1. Phylogenetic relationships among chloroplast genomes of thirty-nine rice accessions. Tree topology based on MrBayes software (branch labels represent probability percentage). 
Considering the variations identified, all thirty-nine rice accessions were sorted into three main groups: (1) Indica, (2) Japonica and (3) Basmati. As expected, the highest number of polymorphisms among the species studied ( 255 bases in 76 variant positions) was found in the Indica group, 11 accessions and 3 Iraqi varieties; within 76 variants, there was only one variation (1-bp deletion at position of 75990 $\mathrm{bp}$ ) between indica and aus accessions. While the second largest number of variations (55 bases within 21 variant positions) was within the Basmati group, 8 accessions and one Iraqi variety. Part of the Basmati group, 4 accessions, showed unique polymorphisms (2 variable bases (SNPs) within 2 variant positions), three accessions were from Pakistan IRIS_313-8656, IRIS_313-11026, and IRIS_313-11021) and one from Iran (CX104). As expected, the Japonica group, 13 accessions along with the reference (O. sativa sub sp. japonica Nipponbare "GenBank: GU592207.1") and one Iraqi variety, possessed the lowest number of polymorphisms (13 bases within 10 variant positions) (Table S4). Most of the polymorphisms in the Japonica group belonged to only four accessions from tropical japonica (TrpJ) subpopulation, including CX352, IRIS_313-10073, CX243, and IRIS_313-11248.

Furthermore, a heat-map was drawn according to the number of variable bases (Table S5); in this map, the two main clusters, Indica and Japonica, were clearly distinguished, whereas the Basmati group was comprised within the Japonica group. Within the Japonica group two individuals, 24:IRIS_313-11479 and 27:IRIS_313-11248, clearly showed the greatest distances among the rice accessions. This cluster surprisingly also included two individuals, 33:IRIS_313-10718 and 34:B009, from the aus and indica ecotype, respectively. There were no variable bases between a number of pairs (dark red in Table S5) such as (3:IRIS_313-11152 and 4:IRIS_313-9505), (9:CX126 and 11:CX37), (9:CX126 and 13:CX25), (17:IRIS_313-10073, and 18:CX243), (19:Ref-GU592207.1 and 20:IRIS_313-11153), (20:IRIS_313-11153 and 21:IRIS_313-10373); and (30:IRIS_313-8656, 31:IRIS_313-11026 and 37:CX104); whereas the highest number of variable bases, 260 bases, was found between (14:CX227 and 24:IRIS_313-11479) (dark green in Table S5). The smallest number of variable bases between Iraqi varieties and other domesticated rice accessions were 1, 3, 1, 6 and 4 bases, those bases were between Iraqi varieties: Amber33, Furat, Yasmin, Buhooth1, and Amber al-Baraka, and the following accessions: 28:IRIS_313-10670, 12:CX10, 3:IRIS_313-11152, 20:IRIS_313-11153 and 5:IRIS_313-10549, respectively (Table S5).

\subsection{Phylogenetic Analysis of the Nuclear Genome}

Within a group of thirty-nine rice accessions, the multiple alignment of 916 concatenated nuclear genes was 621,012 bp in length; the minimum and maximum lengths were 616,099 and 616,393 bp, respectively (Table 3). The nuclear phylogenies using two different methods showed that the two main clusters, Indica and Japonica, and further five sub-clusters were based upon their ecotype, indica, aus, tropical japonica, temperate japonica and basmati (Figure 2). Unlike the results of the chloroplast phylogeny, the accessions of indica, and aus ecotypes were represented by two well-resolved subclades within the Indica clade. The Iraqi varieties, Furat and Yasmin, were found in the indica subclade while the rest of the Iraqi collection was grouped in the Japonica clade, where Amber33 acted as a sister to all the basmati varieties within the basmati subcluster, which included all accessions with the basmati ecotype. Buhooth1 was part of the temperate japonica subcluster that comprised accessions from the temperate japonica ecotype and the reference, O.s japonica cv. Nipponbare. Amber al-Baraka was a sister to both the Indica and Japonica clades; however, Geneious Tree Builder showed that it was close to the Indica clade, while MrBayes suggested that Amber al-Baraka was closer to the Japonica clade. 


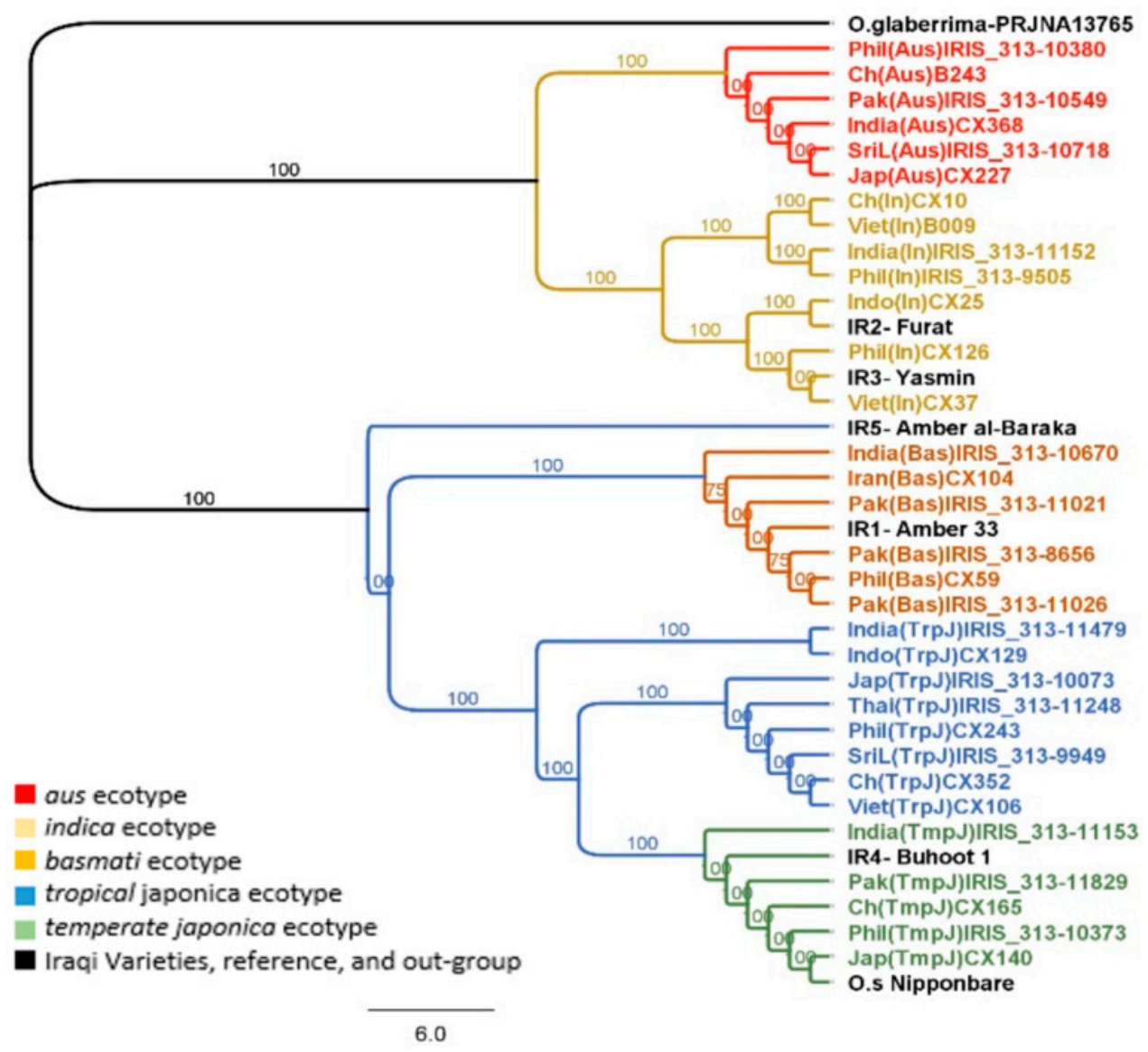

Figure 2. Evolutionary relationships among the multiple alignment of 916 concatenated nuclear genes of domesticated rice. Tree topology based on MrBayes software (branch labels represent probability percentage).

\section{Discussion}

Rice phylogeny has been extensively studied as a better understanding of the evolutionary relationships among rice species is critical for rice breeding programmes as well as comparative genomics studies. Recent advances in next-generation DNA sequencing (NGS) have improved the phylogenetic reconstruction of any plant species including Oryza. In this study, both plastid and nuclear genomes were assembled using NGS reads (whole genome DNA sequencing) to identify the phylogenetic relationships among Iraqi rice varieties and other accessions. According to Sims et al. [27], the accuracy of a genome assembly using NGS reads depends on many factors including sequencing depth (coverage) and the accuracy of the assembly pipeline. Therefore, even after trimming, the sequence coverage of the sequenced and downloaded accessions (Table S1) was enough to ensure coverage of all the chloroplast and most of the nuclear genome, thereby guaranteeing a high-quality assembly.

A dual pipeline was applied to the assembly of the chloroplast genome in this study; this pipeline consisted of two procedures, mapping assembly (MA), and de novo assembly $(d \mathrm{~A})$. The comparison between the sequence of de novo and mapping consensus showed no significant differences in terms of the number of variations. Interestingly, the variety Yasmin showed no difference in both approaches with regard to a number of variations (Table S3), but the length of the consensuses was different; this observation indicates that even when the number of copies of an insertion or deletion was similar, the number of bases that were inserted or deleted was diverse. Therefore, in agreement with an earlier study [28], a manual-curation step was critical in resolving any conflicts by reference to the reads. A pipeline of nuclear genes assembly was also developed in this study. This pipeline involved 
multiple tools on the CLC Genomics Workbench, unlike a previous study [25] that used different software packages to assemble the nuclear genes for phylogenetic analysis at the nuclear genome level. The number of genes selected to represent the nuclear genome in the phylogenetic analysis was only 916 genes with a length of $621,012 \mathrm{bp}$, considerably lower than that reported previously [25].

Phylogenetic analysis of the chloroplast genome sorted the thirty-nine rice accessions into two main clades, an Indica clade and a Japonica clade (Figure 1). The Indica clade (In) included most individuals under the indica and the aus ecotypes except for two accessions. Accessions from indica and aus ecotypes were not clearly distinct but were placed together in one clade; this was confirmed by the results of genetic polymorphism analysis that showed only one variation (1-bp deletion at the position of 75,990 bp; Table S4) between the indica and aus accessions. The Japonica clade (Jap) contained two subclades, the main Japonica clade (Jap) which included all individuals from the two japonica subpopulations, and the Basmati clade (Bas) that included all basmati accessions as well as the individuals excluded from the first clade (Indica). Moreover, the presence of accessions from aus ecotype in the Indica clade as well as in the Basmati subclade within Japonica clade agrees with earlier outcomes [18] which indicated that the two different ecotypes, indica and japonica, might be involved in the origins of the maternal genome in two Korean aus landrace rices. This also agrees with the conclusion made by Civán et al. [29]. which suggested that aromatic rice resulted from a hybridization between japonica and aus. Analysis of genetic polymorphisms at the chloroplast genome level revealed that the most abundant variation types were SNPs, $57 \%$ of 85 variants (Table 4 ). This analysis also showed 255 nucleotide differences within 76 variant positions between the O.sativa spp. indica and the japonica reference (GU592207) in agreement with the previous studies of Brozynska et al. [22] and Wambugu et al. [28].

Table 4. Summary of the number and types of variants in the chloroplast-genomes of thirty-nine domesticated rice-accessions.

\begin{tabular}{|c|c|c|c|c|c|}
\hline Variation Type & SNPs & MNPs & Del & Ins & Total \\
\hline Indica & 37 & 5 & 10 & 9 & 61 \\
\hline Japonica & 1 & 0 & 1 & 1 & 3 \\
\hline Basmati & 4 & 1 & 0 & 1 & 6 \\
\hline Indica \& Basmati & 3 & 2 & 3 & 0 & 8 \\
\hline Indica and Basmati and Japonica & 3 & 0 & 1 & 3 & 7 \\
\hline Total & 48 & 8 & 15 & 14 & 85 \\
\hline
\end{tabular}

At the nuclear genome level, the phylogenetic analysis using two different approaches sorted accessions from indica and aus ecotypes into two completely independent subclades within the Indica clade, unlike the result of the chloroplast phylogeny, whilst the second clade was a Japonica clade which included three sub-clusters tropical japonica, temperate japonica, and basmati (Figure 2). Accordingly, the findings of the evolutionary relationship based on nuclear and chloroplast data in the current study aligned with an earlier study by Garris et al. [30] which reported that the closest evolutionary relationships were between indica and aus groups, and among the tropical japonica, temperate japonica, and aromatic groups. In general, in the present study, the phylogenetic analysis at both genome levels, chloroplast and nuclear, showed relatively comparable evolutionary history patterns with insignificant differences at the end of clades, unlike other studies that recorded significant differences in evolutionary history pattern using both chloroplast and nuclear genomes (regardless of plant materials) [23-25,29]. Furthermore, the phylogenetic trees of both genomes, chloroplast and nuclear, constructed using different methodologies, were highly compatible. However, Amber al-Baraka showed slightly different relationships at the level of the nuclear genome according to the method used; where Geneious Tree Builder software placed Amber al-Baraka close to the Indica clade whereas Amber al-Baraka was closer to the Japonica clade and distant from the Indica clade by MrBayes software. This was unexpected and requires further investigation. 
The phylogenetic analysis of both the chloroplast and nuclear genomes indicated that Amber33, Furat and Yasmin, and Buhooth1 belonged to basmati, indica and japonica ecotypes, respectively. Our results supported that Buhooth1 is an improved cultivar, where the nuclear phylogenies showed a divergent relationship to those deduced from the chloroplast genomes, analogous to temperate japonica subpopulation and tropical japonica subpopulation, respectively. Furat and Yasmin were introduced to Iraq from Vietnam [4], this was obvious by the results of phylogenetic analysis of the nuclear genome, but their chloroplast genome was closely related to accessions from China, India and Philippines. This may be explained by the breeding history of the genotype.

In this study, Amber33, which is local Iraqi variety, was placed in the basmati ecotype group as a sister of cultivars from Pakistan and India by analysing the evolutionary relationship at both levels of the genome. Based on distance analysis, the number of differences in the chloroplast genome between Amber33 and all accessions within the Basmati subclade was in the following order: 28:IRIS_313-10670 O (1 bp), 35:CX59 (1 bp), 30:IRIS_313-8656 (3 bp), 31:IRIS_313-11026 (3 bp), 37:CX104 (3 bp), 33:IRIS_313-10718 (3 bp), 32:IRIS_313-11021 (5 bp), 34:B009 (6 bp) (Table S5); it can be accordingly concluded that Amber33 is closely related to accession from India which is visibly reflected in the observed phylogenetic tree (Figure 1). This confirms the popular tradition that says that the Amber variety was transferred by a group of people who had migrated from India (the Southeast) and settled in southern Iraq a long time ago.

Recently, the term Basmati has been used to indicate a long-grain and high-quality rice, but this name originally refers to aromatic rice because it was derived from the Sanskrit words "Vas" and "Matup" which stand for "aroma" and "ingrained from the beginning", respectively, and then both words were combined making 'Vasmati' which changed to become 'Basmati' later on [31,32]. Therefore, the presence of Amber33 within the Basmati subcluster does not necessarily mean that it is a long grain cultivar; indeed, it is an aromatic medium-grain cultivar. Furthermore, Basmati is a group that can be described basically as the fifth isozyme group identified by Glaszmann [33], and it is closer to the japonica group than the indica $[7,30,34]$; this group is also phenotypically diverse as it includes both long or medium grain, and aromatic or nonaromatic varieties [7]. In many studies, this group is also known as the "aromatic" subpopulation [7,33], but most of the time it is known as "Group V" to avoid confusion. In this study, we refer to this group as "Basmati" according to Wang et al.'s study [26] which is the information resource of the downloaded accessions.

\section{Conclusions}

In the present study, we have assembled the whole chloroplast genome and the nuclear genome of the five Iraqi rice varieties, together with thirty-three domesticated Asian rice, to find the origin of Iraqi varieties, especially Amber33, and to gain insight into the evolutionary relations between Iraqi and domesticated Asian rice varieties. Our results suggest that the possibility of an Indian and/or Pakistani origin for Amber33; to evaluate this hypothesis, further historical biogeographical analyses are required. Moreover, further study on the chloroplast and nuclear genome in Iraqi rice varieties are required to determine the functional genome annotations that might be useful for future rice breeding programmes in Iraq.

\section{Materials and Methods}

\subsection{Plant Materials}

A total of five varieties of Oryza sativa were provided and tested by the Office of Agricultural Research, and Directorate of Seed Testing and Certification, Ministry of Agriculture, Baghdad, IRAQ, respectively. Among these varieties, one variety, Amber33, is local and one of the most highly valued varieties in Iraq because of its fragrance, and two varieties, Furat and Yasmin, were introduced from Vietnam; however, they are successfully cultivated in the central and southern regions of Iraq; while 
the other two, Buhooth1 and Amber al-Baraka, are improved varieties [4]. The plant materials used in this chapter are described in detail in Table 1.

\subsection{Seed Germination and Growth}

About 15 seeds of each individual, a total of 75 seeds, were first dehusked, and then placed in a container with plenty of liquid fertilizer, Flowfeed EX7, that was diluted to half concentration (full concentration is $0.5 \mathrm{~g} / 1 \mathrm{~L}$ ) to break the dormancy phase; this method was the non-heat treatment method. Once the radicle emerged, the germinated seeds were transferred to a petri dish covered with a layer of tissue that was saturated with liquid fertilizer, and planted within three days. All the germination and planting processes were carried out under extremely restricted quarantine conditions in quarantine facilities.

\subsection{DNA Extraction and Sequencing}

After harvesting leaves tissues, total genomic DNA was extracted individually using the modified CTAB protocol described by Furtado [35] with slight modifications. The modifications that were made can be summarised as the following: the mixture of ground plant tissue and nuclear extraction buffer was incubated at $65^{\circ} \mathrm{C}$ for $60 \mathrm{~min}$ with periodic mixing by inverting the tubes every $5 \mathrm{~min}$; as well as the speed and time of centrifuge were increased to $4000 \times g$ and $7 \mathrm{~min}$, respectively, after the steps of protein denaturation and DNA precipitation. However, the most vital modification in the DNA extraction procedure was the exclusion of the mixture of phenol:chloroform:isoamyl alcohol (25:24:1). The quality of DNA was assessed by NanoDrop ${ }^{\mathrm{TM}} 8000$ Spectrophotometers (Thermo Scientific, http://www.nanodrop.com) while the DNA quantity was estimated by agarose gel electrophoresis (1\%, $120 \mathrm{~V}$ for $1 \mathrm{~h}$ ) based on Furtado's study [35].

The whole genomic DNA of Iraqi rice varieties was sequenced by preparing and indexing five PCR-free libraries separately (one library for each variety), then pooling them together and sequencing over a half lane of an Illumina HiSeq 4000 flow-cell at MACROGEN (Seoul, Korea; http://dna.macrogen.com).

\subsection{Data Downloaded for Sequence Comparisons}

Raw sequence reads of 33 domesticated rice accessions were sourced from the Sequence Read Archive (SRA)-NCBI website (https://www.ncbi.nlm.nih.gov/sra) using "Download/Search for Reads in SRA" tool on CLC Genomics Workbench version 11.0.1 (CLC Bio, a QIAGEN Company, Aarhus, Denmark; www.clcbio.com). All of the species, except one, were Asian rice (O. sativa) relatives. O. glaberrima, an African rice, was included as an out-group. All related information such as the sample unique ID, project accession, species, country of origin, and ecotype was obtained from an earlier study [26], as shown in Table 2.

\subsection{Data Processing}

The raw reads of both sequenced and downloaded data were subjected to quality control (QC) analysis using the "Create Sequencing QC Report" tool in the CLC Genomics Workbench, which was used to verify the integrity of the data and determine the appropriate trimming score. The low-quality reads were trimmed at a quality limit of 0.01 and a minimum PHRED score of 25 "Trim Sequences" tool on the CLC.

\subsection{Chloroplast Genome Assembly}

A chloroplast genome of the domesticated rice was assembled and validated using a dual pipeline approach: (1) mapping assembly (MA), and (2) de novo assembly ( $d \mathrm{~A})$ [36]. In the mapping assembly (MA) pipeline, the trimmed reads were mapped against the reference, which is O. sativa sub sp. japonica Nipponbare "GenBank: GU592207.1", using "Map reads to reference" tool at three various 
fraction settings of length-fraction and similarity-fraction (1) 0.8 and $0.8,(2) 1$ and 0.8, and (3) 1 and 0.9 , this step was known as " $\mathrm{R}$ ". Additionally, in an attempt to mend the Cp map, two tools, "InDels and Structural Variants" and "Local Realignment", were applied. This step was named "S". All the analyses of mapping assembly were performed on the CLC Genomics Workbench 11.0.1.

In the de novo assembly pipeline, the Fast " $\mathrm{F}$ " model was used with combinations of Word "W" and Bubble " $\mathrm{B}$ " settings. Contigs generated by de novo were blasted against the Cp reference O. sativa sub sp. japonica Nipponbare "GenBank: GU592207.1" to select the Cp-exclusive contigs, and they were then updated using the "Map Reads to Contigs" tool on the CLC Genomics Workbench 11.0.1. Lastly, the updated contigs were aligned to a reference sequence to recognise overlaps and gaps using Clone Manager Professional 9.0 (www.scied.com). When non-overlapping contigs were produced, supplemental de novo assembly was conducted at various $\mathrm{W}$-and B-settings to plug all gaps by creating additional contigs, and then all the overlapping contigs were subjected to the further analysis.

An additional improvement process was performed on both the mapping and de novo assembly pipelines. The improvement (Imp) process was similar to the mapping assembly (MA) pipeline, repeated twice, Imp-1 and Imp-2, with one difference, the consensus generated from each process would be a reference for the following process. The sequences of both improved $\mathrm{Cp}$ consensus generated by the mapping and de novo improvement processes were compared to identify all mismatches and then were manually corrected by reference to the reads; this step was named "manual-curation" (Figure S2). Eventually, the $\mathrm{Cp}$ sequence of each variety was ready for the phylogenetic analysis.

\subsection{Phylogenetic Analysis}

The consensus chloroplast sequences of the Iraqi rice and the other domesticated rice accessions were used to perform a phylogenetic analysis using Geneious software version 9.1.8 (https://www. geneious.com). The multiple alignment was conducted using the plugin MAFFT Alignment [37] with default parameters; subsequently, to analyse evolutionary relationships; the phylogenetic tree was constructed through software that roots the constructed tree based on the outgroup method: MrBayes [38], and Geneious Tree Builder. The distance between the chloroplast genomes of Iraqi and comparative rice was determined by detecting all the variants using the "variant/SNP detection" tool on Geneious software and then counting the differences (number of bases which are not identical), one of the outputs of the phylogenetic tree construction process.

\subsection{Phylogenetic Analysis of the Nuclear Genome}

An evolutionary relationship analysis at the level of the nuclear genome was undertaken using the CLC Genomics Workbench 11.0.1 and Geneious software version 9.1.8; this analysis started with the nuclear genome assembly (NGA) pipeline (Figure S3). In NGA pipeline, the "Map Reads to Reference" tool was used to map the trimmed reads of the Iraqi rice (Table 1), and the domesticated rice accessions from Asia and Africa (Table 2) against the reference, which is O. sativa sub-spp. Japonica cv Nipponbare "GenBank: IRGSP1.0", applying the following setting: length-fraction of 1 and similarity-fraction of 0.8 . After mapping, the consensus sequence of a whole genome for each variety was extracted using the "Extract Consensus Sequence" tool, and from that, the genome and coding sequence (CDS) tracks were generated by the "Convert to Tracks" tool. By investigating the CDS tracks for all varieties, a subset of 916 genes was identified in all varieties, and then the nucleotide sequences of 916 CDS were separately extracted from the genomes using the "Extract Annotations" tool. At the final stage of the nuclear genome assembly (NGA), all the nucleotide sequences of the 916 CDS selected from each genome were concatenated into a super-matrix of 621,012 bp by the "Join Sequences" tool. The super-matrices of all varieties were then aligned using multiple alignments MAFFT [37] on Geneious at default parameters; the alignment output was used in the following phylogenetic inference. A phylogenetic tree was constructed and rooted using the outgroup methods which are MrBayes [38], and Geneious Tree Builder (https://www.geneious.com); the default tree search settings were applied for both methods. 
Supplementary Materials: The following are available online at http://www.mdpi.com/2223-7747/8/11/481/s1, Figure S1: The results of de novo Assembly on Clone Manager Professional 9.0 software; Figure S2: Illustration of the Chloroplast Genome Assembly Pipeline; Figure S3: Illustration of the Nuclear Genome Assembly Pipeline. Table S1: Summary of the output of sequencing and downloading (Raw Data) and trimming processes; Table S2: Summary of Mapping Assembly process using three different setting of Length fraction and Similarity Fraction; Table S3: Comparison between Mapping and de novo assembly in the number of variations in the chloroplast-genome; Table S4: Details of the polymorphisms identified in aligned chloroplast-genomes using the "variant/SNP detection" tool; Table S5: Distance matrix corresponding to the number of non-identical bases in the sequences of domesticated-rice chloroplast-genomes.

Author Contributions: Conceptualization, R.H. and H.B.; Methodology, H.B and A.F.; Software, A.F. and H.B.; Validation, H.B.; Formal Analysis, H.B.; Investigation, H.B.; Resources, H.B.; Data Curation, H.B.; Writing-Original Draft Preparation, H.B.; Writing-Review \& Editing, R.H., A.F. and H.B.; Visualization, H.B.; Supervision, R.H. and A.F.; Project Administration, A.F.; Funding Acquisition, H.B.

Funding: A PhD scholarship was provided by HCED Iraq program.

Acknowledgments: We would like to acknowledge the HCED Iraq program for providing PhD scholarship and sincerely thank the Office of Agricultural Research and Directorate of Seed Testing and Certification (Ministry of Agriculture, Baghdad, IRAQ) for providing us with the seeds of five Iraqi varieties for phylogenetic analysis. We acknowledge the University of Queensland Research Computing Centre (UQ-RCC) for providing all the computing resources.

Conflicts of Interest: The authors declare no conflict of interest.

Data Availability Statement: All NGS sequence data as raw data was submitted to NCBI at the Sequence Read Archive (SRA) and is available as SRA Submission\# SUB6410326 (under BioProject\# PRJNA576935 and BioSample\# SAMN13014963, SAMN13014964, SAMN13014965, SAMN13014966, and SAMN13014967 represent Amber33, Furat, Yasmin, Buhooth1, and Amber al-Baraka, respectively).

\section{References}

1. Chakravarty, H. Plant Wealth of Iraq: A Dictionary of Economic Plants; Botany Directorate, Ministry of Agriculture \& Agrarian Reform: Baghdad, Iraq, 1976.

2. Chakravarthi, B.K.; Naravaneni, R. SSR marker based DNA fingerprinting and diversity study in rice (Oryza sativa L.). Afr. J. Biotechnol. 2006, 5, 684-688.

3. Rabbani, M.A.; Pervaiz, Z.H.; Masood, M.S. Genetic diversity analysis of traditional and improved cultivars of Pakistani rice (Oryza sativa L.) using RAPD markers. Electron. J. Biotechnol. 2008, 11, 52-61. [CrossRef]

4. Younan, H.Q.; Al-Kazaz, A.A.; Sulaiman, B.K. Investigation of Genetic Diversity and Relationships among a Set of Rice Varieties in Iraq Using Random Amplified Polymorphic DNA (RAPD) Analysis. Jordan J. Biol. Sci. 2011, 4, 249-256.

5. Al-Zahery, N.; Pala, M.; Battaglia, V.; Grugni, V.; Hamod, M.A.; Kashani, B.H.; Olivieri, A.; Torroni, A.; Santachiara-Benerecetti, A.S.; Semino, O. In search of the genetic footprints of Sumerians: A survey of Y-chromosome and mtDNA variation in the Marsh Arabs of Iraq. BMC Evol. Biol. 2011, 11, 288. [CrossRef] [PubMed]

6. Chao, L. Rice Production; Report No. 1081 to the Government of Iraq; FAO: Rome, Italy, 1959.

7. Kovach, M.J.; Calingacion, M.N.; Fitzgerald, M.A.; Mccouch, S.R. The origin and evolution of fragrance in rice (Oryza sativa L.). Proc. Natl. Acad. Sci. USA 2009, 106, 14444-14449. [CrossRef] [PubMed]

8. Al-Judy, N. Detecting of DNA Fingerprints and Genetic Relationship Analysis in Local and Improved Rice (Oryza sativa L.) Varieties in Iraq. Ph.D. Thesis, College of Science-Baghdad University, Baghdad, Iraq, 2004.

9. Al-Kazaz, A.K.A. Sequence Variation and Phylogenetic Relationships Among Ten Iraqi Rice Varieties Using RM171 Marker. Iraqi J. Sci. 2014, 55, 145-150.

10. Patwardhan, A.; Ray, S.; Roy, A. Molecular Markers in Phylogenetic Studies-A Review. J. Phylogenet. Evol. Biol. 2014, 2, 131.

11. Liu, X.; Zhang, H. Advance of molecular marker application in the tobacco research. Afr. J. Biotechnol. 2008, 7, 4827-4831.

12. Sotowa, M.; Ootsuka, K.; Kobayashi, Y.; Hao, Y.; Tanaka, K.; Ichitani, K.; Flowers, J.; Purugganan, M.; Nakamura, I.; Sato, Y.-I.; et al. Molecular relationships between Australian annual wild rice, Oryza meridionalis and two related perennial forms. Rice 2013, 6, 26. [CrossRef]

13. Nock, C.J.; Waters, D.L.E.; Edwards, M.A.; Bowen, S.G.; Rice, N.; Cordeiro, G.M.; Henry, R.J. Chloroplast genome sequences from total DNA for plant identification. Plant Biotechnol. J. 2011, 9, 328-333. [CrossRef] 
14. Straub, S.C.K.; Parks, M.; Weitemier, K.; Fishbein, M.; Cronn, R.C.; Liston, A. Navigating the tip of the genomic iceberg: Next-generation sequencing for plant systematics. Am. J. Bot. 2012, 99, 349-364. [CrossRef] [PubMed]

15. Mcpherson, H.; Van Der Merwe, M.; Delaney, S.K.; Edwards, M.A.; Henry, R.J.; Mcintosh, E.; Rymer, P.D.; Milner, M.L.; Siow, J.; Rossetto, M. Capturing chloroplast variation for molecular ecology studies: A simple next generation sequencing approach applied to a rainforest tree. BMC Ecol. 2013, 13, 8. [CrossRef] [PubMed]

16. Waters, D.L.E.; Nock, C.J.; Ishikawa, R.; Rice, N.; Henry, R.J. Chloroplast genome sequence confirms distinctness of Australian and Asian wild rice. Ecol. Evol. 2012, 2, 211-217. [CrossRef] [PubMed]

17. Yang, J.-B.; Tang, M.; Li, H.-T.; Zhang, Z.-R.; Li, D.-Z. Complete chloroplast genome of the genus Cymbidium: Lights into the species identification, phylogenetic implications and population genetic analyses. BMC Evol. Biol. 2013, 13, 84. [CrossRef] [PubMed]

18. Tong, W.; He, Q.; Wang, X.Q.; Yoon, M.Y.; Ra, W.H.; Li, F.; Yu, J.; Oo, W.H.; Min, S.K.; Choi, B.W.; et al. A chloroplast variation map generated using whole genome re-sequencing of Korean landrace rice reveals phylogenetic relationships among Oryza sativa subspecies. Biol. J. Linn. Soc. 2015, 115, 940-952. [CrossRef]

19. Tong, W.; Kim, T.-S.; Park, Y.-J. Rice Chloroplast Genome Variation Architecture and Phylogenetic Dissection in Diverse Oryza Species Assessed by Whole-Genome Resequencing. Rice 2016, 9, 57. [CrossRef]

20. Parks, M.; Cronn, R.; Liston, A. Increasing phylogenetic resolution at low taxonomic levels using massively parallel sequencing of chloroplast genomes. BMC Biol. 2009, 7, 84. [CrossRef]

21. Moore, M.J.; Pamela, S.S.; Charles, D.B.; Burleigh, J.G.; Douglas, E.S. Phylogenetic analysis of 83 plastid genes further resolves the early diversification of eudicots. Proc. Natl. Acad. Sci. USA 2010, 107, 4623-4628. [CrossRef]

22. Brozynska, M.; Omar, E.S.; Furtado, A.; Crayn, D.; Simon, B.; Ishikawa, R.; Henry, R.J. Chloroplast Genome of Novel Rice Germplasm Identified in Northern Australia. Trop. Plant Biol. 2014, 7, 111-120. [CrossRef]

23. Huang, X.; Kurata, N.; Wang, Z.X.; Wang, A.; Zhao, Q.; Zhao, Y.; Liu, K.; Lu, H.; Li, W.; Guo, Y.; et al. A map of rice genome variation reveals the origin of cultivated rice. Nature 2012, 490, 497-501. [CrossRef]

24. Kim, H.; Jeong, E.; Ahn, S.-N.; Doyle, J.; Singh, N.; Greenberg, A.; Won, Y.; Mccouch, S. Nuclear and chloroplast diversity and phenotypic distribution of rice (Oryza sativa L.) germplasm from the democratic people's republic of Korea (DPRK North Korea). Rice 2014, 7, 7. [CrossRef] [PubMed]

25. Brozynska, M.; Copetti, D.; Furtado, A.; Wing, R.A.; Crayn, D.; Fox, G.; Ishikawa, R.; Henry, R.J. Sequencing of Australian wild rice genomes reveals ancestral relationships with domesticated rice. Plant Biotechnol. J. 2017, 15, 765-774. [CrossRef] [PubMed]

26. Wang, W.; Mauleon, R.; Hu, Z.; Chebotarov, D.; Tai, S.; Wu, Z.; Li, M.; Zheng, T.; Fuentes, R.R.; Zhang, F.; et al. Genomic variation in 3,010 diverse accessions of Asian cultivated rice. Nature 2018, 557, 43-49. [CrossRef] [PubMed]

27. Sims, D.; Ian, S.; Nicholas, E.I.; Andreas, H.; Chris, P.P. Sequencing depth and coverage: Key considerations in genomic analyses. Nat. Rev. Genet. 2014, 15, 121. [CrossRef]

28. Wambugu, P.W.; Brozynska, M.; Furtado, A.; Waters, D.L.; Henry, R.J. Relationships of wild and domesticated rices (Oryza AA genome species) based upon whole chloroplast genome sequences. Sci. Rep. 2015, 5, 13957. [CrossRef]

29. Civáň, P.; Craig, H.; Cox, C.J.; Brown, T.A. Three geographically separate domestications of Asian rice. Nat. Plants 2015, 1, 15164. [CrossRef]

30. Garris, A.J.; Tai, T.H.; Coburn, J.; Kresovich, S.; Mccouch, S. Genetic Structure and Diversity in Oryza sativa L. Genetics 2005, 169, 1631-1638. [CrossRef]

31. Ahuja, S.C.; Panwar, D.V.S.; Uma, A.; Gupta, K.R. Basmati Rice: The Scented Pearl, Hisar, Directorate of Publications; CCS Haryana Agricultural University: Hisar, India, 1995.

32. Akram, M. Aromatic rices of Pakistan: A review. Pak. J. Agric. Res. 2009, 22, 154-160.

33. Glaszmann, J.C. Isozymes and classification of Asian rice varieties. Tag. Theor. Appl. Genet. Theor. Angew. Genet. 1987, 74, 21-30. [CrossRef]

34. Caicedo, A.L.; Williamson, S.H.; Hernandez, R.D.; Boyko, A.; Fledel-Alon, A.; York, T.L.; Polato, N.R.; Olsen, K.M.; Nielsen, R.; McCouch, S.R.; et al. Genome-wide patterns of nucleotide polymorphism in domesticated rice. PLoS Genet. 2007, 3, e163. [CrossRef]

35. Furtado, A. DNA extraction from vegetative tissue for next-generation sequencing. Methods Mol. Biol. 2014, 1099, 1-5. [PubMed] 
36. Moner, A.M.; Furtado, A.; Henry, R.J. Chloroplast phylogeography of AA genome rice species. Mol. Phylogenet. Evol. 2018, 127, 475-487. [CrossRef] [PubMed]

37. Katoh, K.; Misawa, K.; Kuma, K.; Miyata, T. MAFFT: A novel method for rapid multiple sequence alignment based on fast Fourier transform. Nucleic Acids Res. 2002, 30, 3059-3066. [CrossRef] [PubMed]

38. Huelsenbeck, J.P.; Ronquist, F. MRBAYES: Bayesian inference of phylogenetic trees. Bioinformatics 2001, 17, 754-755. [CrossRef]

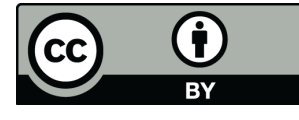

(C) 2019 by the authors. Licensee MDPI, Basel, Switzerland. This article is an open access article distributed under the terms and conditions of the Creative Commons Attribution (CC BY) license (http://creativecommons.org/licenses/by/4.0/). 